\title{
Vegetation structure and floristic composition (Case study: Mala Galeh Protected area, Fars Province, Iran)
}

\author{
Struktur vegetasi dan komposisi bunga (Studi kasus: Kawasan Lindung Mala Galeh, Provinsi \\ Fars, Iran)
}

Leila Moradipour ${ }^{\mathrm{a}}$, Hasan Pourbabaei ${ }^{\mathrm{b}}$, Ahmad Hatamic $^{\mathrm{c}}$

${ }^{\text {a }}$ PHD Student Gorgan University of Agricultural Sciences and Natural Resources

${ }^{b}$ Department of forestry, Faculty of Natural Resources, University of Guilan, Iran [098 13443233599]

${ }^{\mathrm{c}}$ Research Center of Agricultural and Natural Resources of Shiraz, Iran [098 07137209332]

\section{Article Info:}

Received: 05 - 10 - 2019

Accepted: $31-05-2020$

\section{Keywords:}

Conservation plant, FIV, Iran, life form, SIV

Corresponding Author:

Hasan Pourbabaei

Department of forestry, Faculty

of Natural Resources, University

of Guilan;

Email:

moradiporleila@gmail.com

\begin{abstract}
Identifying flora of each region is fundamental for accomplishing other pure and applied researches in biology. Especially, in the ecological conditions of protected area of Male Gale. Data were collected in 96 sampling plots using systematic-random method. The size of sampling plot was $(20 \times$ 50) $m$ for the tree and shrub species, and $(8 \times 8) m$ for herbaceous species. In this study area, 162 species, 122 genera, and 43 families were identified. The largest families were Asteraceae (26 species) and Fabaceae (25 species). The frequency of Asteraceae may be due to grazing in some areas of the region. The life-form spectrum includes Hemichryptophytes (14/01\%), Therophytes (65/4\%), Cryptophytes (76/9\%), Chamaephytes (7.1\%), and Phanerophytes (5.8\%). The abundance of Therophytes and Asteraceae family is referred to the destruction of forests in the study area. The highest value of the SIV tree and shrub species layer belong to Quercus brantii species and Ziziphus Mummularia. The highest value of the FIV herbaceous layer belongs to Asteraceae family. The Species Important Value (SIV) of vegetation cover indicated that trees, shrubs species, and herbaceous species had geometric distribution, broken stick model, and lognormal distribution in this area.
\end{abstract}

How to cite (CSE Style $8^{\text {th }}$ Edition):

Moradipour L, Pourbabaei H, Hitami A. 2020. Vegetation structure and floristic composition (Case study: Mala Galeh Protected area, Fars Province, Iran). JPSL 10(3): 533-544. http://dx.doi.org/10.29244/jps1.10.3.533-544.

\section{INTRODUCTION}

Iran with an area of $1648000 \mathrm{~km}^{2}$ is located in the south west of the continent of Asia (Moradkhani and Milan, 2015). Mountainous status of Iran and environmental factors such as: (climate, topography, soil) caused many species diversities in different regions, so, it has been the attention of researchers. Zagros Mountains is stretched from south-west to northwest of Iran and the altitude is about 1000-4300 m (Moradipour et al., 2018; Noroozi et al., 2008). This ecosystem has been divided into three parts: northern, central and southern Zagros (Valipour et al., 2009). The number of plant species known in Iran is about 7600. The definition of protected areas based on IUCN (1976): Lands which, owing to their strategic value to the conservation of the nation's natural resources, are to be managed in a manner what will prevent degradation or, if already degraded, can be rehabilitated primarily through natural processes. Protection, management and restoration of plant and animal 
life and the maintenance of the natural state will be afforded the highest priority in providing conditions conducive to the regeneration and amelioration of habitats and species.

Identifying flora of each region is fundamental for accomplishing other pure and applied researches in biology (Rios and Recio, 2005; Heinrich et al., 2004). Identify plants contribute greatly to the conservation and protection of the environment (Vaseghi et al., 2007). Furthermore, with respect to the environmental disturbances like global warming, climate changes and human impact which change the number of plant species, (Parmesan, 2007; Parmesan and Yohe, 2003; Perry et al., 2005; Körner and Basler, 2010). In the protected area of Malé Galle protected unique ecological and climatic conditions make it a remarkable habitat for the floristic studies. So, winter and rural rangelands comprise a large fraction of Male Galeh area. People in this region are highly dependent on rangelands; they use plants as sources for food, medicine, livestock production and etc. (Negahdarsaber et al., 2017). Based on Raunkiaer's life-forms, plant species can be grouped into different life-forms classes based on structural and functional similarities (Smith, 1913; Sarmiento and Monasterio, 1983). Phytochorya study in a region is the base of ecological studies for better management of area (Moradkhani and Milan, 2015). In addition, Floristic survey of an area is an essential tool for conservation of biodiversity (Noroozi et al., 2008).

Nowadays, many studies have been doing about this subjects in Iran and the world, such as: floristic study of Firuzeh watershed in north of khorasan province (Asaadi, 2009), study of life form in Sepidan, Fars Province, Iran (Negahdarsaber et al., 2017). Study of life form and chorology of plants in Jozak - Chaminbid area, north Khorassan Province, Iran (Nadaf et al., 2017). Vegetation basically is described by density, DBH and frequency parameters (Razavi et al., 2012). SIV (Species importance value) as an index covers all of these parameters (frequency, abundance, basal area) together for the determination of distribution and frequency patterns of species as well as judgments about ecological conditions that have more significance and for families FIV (Family Importance Value) used. Researchers proposed some models for the determination of frequency patterns. These distributions included geometric series, normal logarithmic and broken stick methods (Magurran, 2003). The purpose of this research was Study on Floristic Composition, to use SIV and FIV for the assessment of distribution of abundance of species.

\section{MATERIAL AND METHODS}

\section{Study Area}

The present study was carried out in the protected area of Malé Galle forest in the end of Komaresorkhi, Fars Province. This area is located at South Zagros, Fars province. Which is about 300 ha. The study area is located between $29^{\circ} 15^{\prime} 57^{\prime \prime}-30^{\circ} 36^{\prime} 5^{\prime \prime} \mathrm{N}$ latitude and $51^{\circ} 33^{\prime} 37^{\prime \prime}-52^{\circ} 25^{\prime} 56^{\prime \prime}$ E longitude, in the southwest part of Iran. This region has semiarid temperate climate with the average annual precipitation of $593.4 \mathrm{~mm}$ and the mean annual temperature of $15.6^{\circ} \mathrm{C}$. The dry season is started from beginning of May through October (seven months) (Moradipour et al., 2018).

\section{Data Collection and Analysis}

Sampling vegetation was conducted in spring 2016. For this purpose, a random-systematic with $(150 \times 150) \mathrm{m}$ inventory grid was used to establish 96 plots. The size of sampling plots was $1000 \mathrm{~m}^{2}$ for the tree and shrub species, and $64 \mathrm{~m}^{2}$ for herbaceous species. Percent cover of herbaceous and the crown cover of tree species were recorded. Chorotypes and lifeforms of each species were determined (Negahdarsaber et al., 2017). Density, abundance, frequency, basal area and species importance value (SIV) for each species have been calculated. The equations used are given as below (Pourbabaei et al., 2013): 
SIV for tree layers $=$ relative frequency + relative density + relative dominance

Relative frequency $=($ number of plots containing a species $\times 100) /$ total plots

Relative density $=$ (number of individuals of a species $\times 100) /$ total number of individuals of all species

Relative dominance $=($ basal area of a species $\times 100) /$ total basal area of all species

SIV for shrub layers $=$ relative frequency + relative density

The Family Importance Value (FIV) was calculated as follows:

FIV $\quad=$ relative density + relative diversity + relative dominance

Relative densit $\quad=$ (number of individuals of the species $\times 100$ )/total number of individuals in the sample

Relative diversity $=$ (number of species in the family $\times 100$ )/total number of species in the sample

Relative dominance $=($ basal area of the family $\times 100) /$ total basal area in the sample

\section{HASIL DAN PEMBAHASAN}

\section{Results}

In the study area, 162 species were recorded belonging to 122 genera, and 43 families. 74 species of 162 plant species were indicator species. The phytogeographical elements include Irano-Turanian (42/5\%), IranoTuranian-Mediterranean (17/9\%), Irano-Turanian-Sahara Sindian (13/7\%). So, Irano-Turanian was the most dominant Chorotypes (Table 1).

Table 1 List of, species, family, life form and chorotypes in the study area. chorotypes (IT: Irano-Turanian;

ES: European-Siberian; M: Mediterranean; Cosm: Cosmopolite; SS: Sahara-Sindian), Life forms (Th:

Therophyte; H: Hemicryptophyte; Ch: Chamaephyte; Cr: Cryptophyte; Ph: Phanerophyte)

\begin{tabular}{|c|c|c|c|c|}
\hline Family and Scientific name & $\begin{array}{l}\text { Life- } \\
\text { form }\end{array}$ & Chorotype & $\begin{array}{c}\text { Indicator } \\
\text { species }\end{array}$ & $\begin{array}{l}\text { Medicinal } \\
\text { plant }\end{array}$ \\
\hline \multicolumn{5}{|l|}{ Asteraceae } \\
\hline Carduus arabicus Jacq ex. Murray & $\mathrm{T}$ & $\mathrm{ES}, \mathrm{M}$ & $*$ & \\
\hline Gymnarrhena micrantha Desf. & $\mathrm{He}$ & IT,SS & & \\
\hline Reichardia orientalis (L.) Hochreutiner & $\mathrm{T}$ & IT & & \\
\hline Artemisia aucherii Boiss. & $\mathrm{Ch}$ & IT & $*$ & $*$ \\
\hline Cymbolaena griffithii & $\mathrm{T}$ & IT & & \\
\hline Achillea wilhelmsii C. Koch & $\mathrm{He}$ & IT & & $*$ \\
\hline Atractylis cancellata $\mathrm{L}$. & $\mathrm{T}$ & IT,M & $*$ & \\
\hline Calendula Persica C. A. Mey. & $\mathrm{T}$ & IT,M & & \\
\hline Outreya carduiformis & $\mathrm{T}$ & IT,SS & & \\
\hline Crepis kotschyana (Boiss.) Boiss. & $\mathrm{T}$ & IT,M & $*$ & \\
\hline Crepis sancta (L.) Babcock & $\mathrm{T}$ & IT,SS & $*$ & \\
\hline Centaurea bruguieriana (DC.) Hand. Mzt & $\mathrm{T}$ & IT, SS & & \\
\hline Koelpinia tenuissima Pavl. \&Lipsch. & $\mathrm{T}$ & IT & $*$ & \\
\hline Lactucaserriola $\mathrm{L}$. & $\mathrm{He}$ & IT,ES,M & $*$ & \\
\hline Launea procumbens (Roxb.) & $\mathrm{He}$ & IT & & \\
\hline \multicolumn{5}{|l|}{ Ramayya\&Rajagopal. } \\
\hline Senecio glaucus L. & $\mathrm{T}$ & IT,M,SS & & \\
\hline Tragopon longirostris Bisch. & $\mathrm{T}$ & IT & & \\
\hline Gundelia tournefortii L. & $\mathrm{He}$ & IT & & \\
\hline Anthemis altissima $\mathrm{L}$. & $\mathrm{T}$ & IT & $*$ & $*$ \\
\hline Anthemis persica Boiss. & $\mathrm{T}$ & IT & & $*$ \\
\hline Anthemis austro - iranicaRech. f. & $\mathrm{T}$ & IT & $*$ & $*$ \\
\hline
\end{tabular}




\begin{tabular}{|c|c|c|c|c|}
\hline Anthemis haussknechtiiBoiss. \&Reut. & $\mathrm{T}$ & IT & & $*$ \\
\hline Anthmis tinctoria & $\mathrm{T}$ & IT,M & $*$ & * \\
\hline Centaur eaintricata Boiss. & $\mathrm{Ch}$ & IT & & \\
\hline Filago desertorum Pomel & $\mathrm{T}$ & IT, ES, SS & & \\
\hline $\begin{array}{l}\text { Picnomon acarna }(\text { L.) Cass. } \\
\text { Apiaceae }\end{array}$ & $\mathrm{He}$ & IT,M & & \\
\hline Falcaria vulgaris Bernh. & $\mathrm{He}$ & IT, SS & & \\
\hline Pimpinella barbata (DC.) Boiss & $\mathrm{T}$ & IT,SS & $*$ & \\
\hline Scandixpecten - veneris L. & $\mathrm{T}$ & IT & $*$ & \\
\hline Torilis leptophylla (L.) Reichenb. & $\mathrm{T}$ & $\mathrm{ES}, \mathrm{IT}, \mathrm{M}$ & & \\
\hline Bupleurum croceum Fenzl & $\mathrm{T}$ & IT & & \\
\hline Lagoecia cuminoides $\mathrm{L}$. & $\mathrm{T}$ & IT & $*$ & \\
\hline Oliveria decumbens Vent. & $\mathrm{T}$ & IT & $*$ & $*$ \\
\hline Aniso sciadiumorientale DC. & $\mathrm{T}$ & IT & & \\
\hline $\begin{array}{l}\text { Eryngium billardieriF.Delaroche } \\
\text { Araceae }\end{array}$ & $\mathrm{He}$ & IT & & \\
\hline $\begin{array}{l}\text { Biarum carduchorum } \\
\text { Anacaridaceae }\end{array}$ & $\mathrm{Cr}$ & IT & & \\
\hline Pistacia khinjuk Stocks & $\mathrm{P}$ & IT & $*$ & $*$ \\
\hline $\begin{array}{l}\text { Pistacia atlantica Desf. } \\
\text { Aizoaceae }\end{array}$ & $\mathrm{P}$ & IT & $*$ & $*$ \\
\hline $\begin{array}{l}\text { Aizoon canariense } \mathrm{L} \text {. } \\
\text { Aceraceae }\end{array}$ & $\mathrm{T}$ & IT,M & & \\
\hline $\begin{array}{l}\text { Acer monspessulanum L. subsp. Cinerascens } \\
\text { (Boiss.)Yaltri } \\
\text { Amaryllidaceae }\end{array}$ & $\mathrm{P}$ & IT & $*$ & \\
\hline $\begin{array}{l}\text { Ixiolirion tataricum (Pall.) Herb. } \\
\text { Brassicaceae }\end{array}$ & $\mathrm{Cr}$ & IT, SS, ES & & \\
\hline Erucaria hispanica (L.) Druce & $\mathrm{T}$ & IT, M & & \\
\hline Allysuminflatum Nyarady. & $\mathrm{T}$ & IT, M & $*$ & \\
\hline Bisculella didyma $\mathrm{L}$ & $\mathrm{T}$ & IT, M & $*$ & \\
\hline Brassica deflexa Boiss. & $\mathrm{T}$ & IT & $*$ & \\
\hline Capsella bursa-pastoris (L.) Medicus & $\mathrm{T}$ & IT, M, SS & & $*$ \\
\hline Matthiola longipetala (Vent.) DC. & $\mathrm{T}$ & IT, M & & \\
\hline Sinapis aucheri (Boiss.) O. E. Schulz & $\mathrm{T}$ & IT & $*$ & \\
\hline Cardaria draba (L.) Desv. & $\mathrm{T}$ & COSM & $*$ & $*$ \\
\hline Clypeola dicotomaBoiss. & $\mathrm{T}$ & IT & $*$ & \\
\hline Euclidium syriacum $(\mathrm{L}.) \mathrm{R}$. Br. & $\mathrm{T}$ & IT & & \\
\hline $\begin{array}{l}\text { Boraginaceae } \\
\text { Arnebiadecumbens (Vent.) Coss. \& Kral }\end{array}$ & $\mathrm{T}$ & IT,SS & & \\
\hline $\begin{array}{l}\text { Cistaceae } \\
\text { Helianthemum aegyptiacum (L.) Miller }\end{array}$ & $\mathrm{T}$ & IT,M,SS & $*$ & \\
\hline $\begin{array}{l}\text { Helianthemum europaeum } \mathrm{L} \text {. } \\
\text { Crassulaceae }\end{array}$ & $\mathrm{T}$ & IT,M,SS & & \\
\hline Umbilicus intermedicusBoiss. & $\mathrm{Ge}$ & IT,M & & \\
\hline $\begin{array}{l}\text { Sedum rubens } \mathrm{L} \\
\text { Cryptogrammaceae }\end{array}$ & $\mathrm{T}$ & IT, M & $*$ & \\
\hline
\end{tabular}




\begin{tabular}{|c|c|c|c|c|}
\hline Onychium melanolepis (Dcne) Kze. & $\mathrm{Cr}$ & IT & & \\
\hline $\begin{array}{l}\text { Convolvulaceae } \\
\text { Convolvulus leiocalycinus Boiss }\end{array}$ & $\mathrm{Ch}$ & IT,SS & & \\
\hline $\begin{array}{l}\text { Convolvulus oxyphyllus Boiss } \\
\text { Chenopodiaceae }\end{array}$ & $\mathrm{Ch}$ & IT, SS & & \\
\hline $\begin{array}{l}\text { Noaea mucronata (Forssk.) Aschers. \& } \\
\text { Schweinf }\end{array}$ & $\mathrm{Ch}$ & IT & & \\
\hline $\begin{array}{l}\text { Dipsacaceae } \\
\text { Scabiosa olivieri Coult } \\
\text { Euphorbiaceae }\end{array}$ & $\mathrm{T}$ & IT & & \\
\hline Euphorbia helioscopia $\mathrm{L}$. & $\mathrm{T}$ & IT & & \\
\hline $\begin{array}{l}\text { Chrozophora tinctoria (L.) Juss. } \\
\text { Fagaceae }\end{array}$ & $\mathrm{T}$ & IT, M & & \\
\hline $\begin{array}{l}\text { Quercus brantii Lindl. } \\
\text { Fumariaceae }\end{array}$ & $\mathrm{P}$ & IT & $*$ & \\
\hline Fumaria bracteosa Pomel & $\mathrm{T}$ & IT & & \\
\hline Fumaria densiflora DC. & $\mathrm{T}$ & IT & $*$ & \\
\hline $\begin{array}{l}\text { Gentianaceae } \\
\text { Gentianao livieri Griseb. } \\
\text { Geraniaceae }\end{array}$ & $\mathrm{He}$ & IT & & \\
\hline Erodium cicularium (L.) Lher. & $\mathrm{T}$ & IT,M,ES & $*$ & \\
\hline Erodium moschatumer (L.) Lher. Ex Aiton & $\mathrm{T}$ & IT,M & $*$ & \\
\hline Geranium rotundifolium $\mathrm{L}$. & $\mathrm{T}$ & IT,M,ES & $*$ & \\
\hline Erodium gruinum (L.) L Her. Ex Aiton. & $\mathrm{T}$ & M & $*$ & * \\
\hline $\begin{array}{l}\text { Lamiaceae } \\
\text { Ajuga astro-iranica } \text { Reth. F. }\end{array}$ & $\mathrm{Ch}$ & IT & $*$ & \\
\hline Marrubium Cuneatum Russel & $\mathrm{He}$ & IT & & \\
\hline Phlomis olivieri Benth. & $\mathrm{He}$ & IT & & \\
\hline Phlomis bruguieri Desf. & $\mathrm{Ch}$ & IT & $*$ & \\
\hline Salvia compressa Vevt. & $\mathrm{He}$ & IT & & $*$ \\
\hline Salvia macrosiphon Bioss. & $\mathrm{T}$ & IT & $*$ & \\
\hline Teucrium polium $\mathrm{L}$. & $\mathrm{He}$ & IT,M & & $*$ \\
\hline Ziziphora tenuir L. & $\mathrm{T}$ & IT & $*$ & * \\
\hline $\begin{array}{l}\text { Lallemantia iberica (Stev.) Fisch. \& C. A. } \\
\text { Mey. }\end{array}$ & $\mathrm{T}$ & IT & $*$ & \\
\hline Lamium amplexicaule $\mathrm{L}$. & $\mathrm{Ch}$ & IT, ES & $*$ & \\
\hline Teucrium orientale $\mathrm{L}$. & $\mathrm{He}$ & IT,SS & $*$ & $*$ \\
\hline Otostegia persica & $\mathrm{Ch}$ & SS & $*$ & * \\
\hline $\begin{array}{l}\text { Liliaceae } \\
\text { Linum strictum } \mathrm{L} \text {. }\end{array}$ & $\mathrm{T}$ & IT & & \\
\hline Gagea tenuifolia (Boiss.) Fomin & $\mathrm{Cr}$ & IT & $*$ & \\
\hline Muscari tenuiflorum Tausch & $\mathrm{Ge}$ & IT & $*$ & \\
\hline Ornithogalum persicum Hausskn. Ex Bornm. & $\mathrm{Cr}$ & IT,M & & \\
\hline Muscari neglectum Guss & $\mathrm{Cr}$ & IT,ES & $*$ & \\
\hline Tulipa stylosa Stapf & $\mathrm{Cr}$ & IT.M & $*$ & \\
\hline Allium stamineum Boiss & $\mathrm{Cr}$ & M & $*$ & \\
\hline Malvaceae & $\mathrm{T}$ & IT, M, SS & & $*$ \\
\hline
\end{tabular}




\begin{tabular}{|c|c|c|c|c|}
\hline \multicolumn{5}{|l|}{$\begin{array}{l}\text { Malva parviflora } \mathrm{L} \text {. } \\
\text { Moraceae }\end{array}$} \\
\hline Ficus johannis Boiss. & $\mathrm{P}$ & IT & & \\
\hline \multicolumn{5}{|l|}{ Plumbaginaceae } \\
\hline Acantholimon asphodelinum Mobayen. & $\mathrm{CH}$ & IT & & \\
\hline $\begin{array}{l}\text { Papaveraceae } \\
\text { Roemeria hybrida (L.) DC. } \\
\text { Fabaceae }\end{array}$ & $\mathrm{T}$ & IT, SS & & \\
\hline Astragalus glaucacanthus Fisch. & $\mathrm{CH}$ & IT & $*$ & \\
\hline Alcea aucheri (Boiss.) Alef. & $\mathrm{He}$ & IT & & \\
\hline Hymenocarpus circinnatus (L.) Savi & $\mathrm{T}$ & M & & \\
\hline Lathyrus inconspicuus $\mathrm{L}$. & $\mathrm{T}$ & IT & & \\
\hline Ononis viscosa $\mathrm{L}$. & $\mathrm{T}$ & IT & & \\
\hline Ebenus stellata Boiss & $\mathrm{Ch}$ & IT & $*$ & \\
\hline Hippocrepis unisiliquosa $\mathrm{L}$. & $\mathrm{T}$ & M & & \\
\hline Lens cyanea (Boiss. and Hohen.) Alef. & $\mathrm{T}$ & IT & & \\
\hline Lens orientalis Boiss. & $\mathrm{T}$ & IT & $*$ & \\
\hline Medicago coronata $(\mathrm{L}$.$) Bartalini$ & $\mathrm{T}$ & IT & & \\
\hline Medicago minima (L.) Bartalini & $\mathrm{T}$ & IT,M,SS,ES & $*$ & $*$ \\
\hline Medicago orbicularis (L.) Bartalini & $\mathrm{T}$ & IT & $*$ & \\
\hline Medicago polymorpha $\mathrm{L}$. & $\mathrm{T}$ & IT.M & $*$ & \\
\hline Medicago rigidula (L.) All. & $\mathrm{pl}$ & IT & & \\
\hline Melilotus sulcatus & $\mathrm{T}$ & IT & & \\
\hline Onobrychis crista-galli (L.) Lam. & $\mathrm{T}$ & IT,SS & & \\
\hline Scorpiurus muricatus L. & $\mathrm{T}$ & $\mathrm{M}$ & & \\
\hline Trifolium campestre Schreb. & $\mathrm{T}$ & IT & $*$ & $*$ \\
\hline Trifolium resupinatum $\mathrm{L}$. & $\mathrm{T}$ & IT & $*$ & \\
\hline Trifolium tomentosum $\mathrm{L}$. & $\mathrm{T}$ & IT & & \\
\hline Trifolium dasyurum C. Presl & $\mathrm{T}$ & ES, IT & & \\
\hline Trigonella monspeliaca $\mathrm{L}$. & $\mathrm{T}$ & IT.M & $*$ & \\
\hline Trigonella elliptica Boiss. & $\mathrm{T}$ & H. IT,SS & $*$ & \\
\hline Vicia sativa $\mathrm{L}$. & $\mathrm{T}$ & IT,ES,M & & \\
\hline Vicia peregrina $\mathrm{L}$. & $\mathrm{T}$ & IT & & \\
\hline $\begin{array}{l}\text { Plantaginaceae } \\
\text { Plantago lagopus L. }\end{array}$ & $\mathrm{He}$ & IT, M, SS & $*$ & \\
\hline Plantago ovata Forssk. & $\mathrm{T}$ & IT,SS,M,ES & $*$ & \\
\hline Plantago psyllium $\mathrm{L}$. & $\mathrm{T}$ & IT,SS,M,ES & $*$ & \\
\hline Plantago coronopus L. & $\mathrm{He}$ & IT, SS & & \\
\hline Plantago bellardi All. & $\mathrm{T}$ & IT, SS & & \\
\hline $\begin{array}{l}\text { Poaceae } \\
\text { Aegilops umbellulata Zhuk. }\end{array}$ & $\mathrm{T}$ & IT & $*$ & \\
\hline Avena fatua $\mathrm{L}$. & $\mathrm{T}$ & IT & $*$ & \\
\hline Bromus danthoniae Trin. & $\mathrm{T}$ & COSM & & \\
\hline Bromus scoparius L. & $\mathrm{T}$ & IT,ES & & \\
\hline Bromus tectorum L. & $\mathrm{T}$ & IT,SS,M,ES & $*$ & \\
\hline $\begin{array}{l}\text { Heteranthelium piliferum (Banks \&Soland.) } \\
\text { Hochst. }\end{array}$ & $\mathrm{T}$ & IT,M & & \\
\hline
\end{tabular}




\begin{tabular}{|c|c|c|c|c|}
\hline Phalaris minor Retz. & $\mathrm{T}$ & IT & & \\
\hline Cynodon dactylon (L.) Pers. & $\mathrm{T}$ & IT,M & & \\
\hline Poa bulbosum $\mathrm{L}$. & $\mathrm{Cr}$ & COSM & $*$ & * \\
\hline Hordeum bulbosa $\mathrm{L}$. & $\mathrm{T}$ & IT & & \\
\hline Lophochloa phleoides (Vill.) Reichenb.. & $\mathrm{T}$ & ES, IT, M, SS & & \\
\hline Poa bulbosa L. & $\mathrm{Cr}$ & ES, IT, M, SS & $*$ & $*$ \\
\hline Poa sinaica steud. & $\mathrm{Cr}$ & IT,M,ES & $*$ & \\
\hline Stipa capensis Thunb. & $\mathrm{Cr}$ & IT,M,SS & & \\
\hline Bromus squarrosa $L$. & $\mathrm{T}$ & IT,SS & & \\
\hline Hordeum glaucum Steud & $\mathrm{T}$ & ES, IT & $*$ & \\
\hline Taeniatherum crinitum (Schreb.) Neveski & $\mathrm{T}$ & IT,M,SS & & \\
\hline $\begin{array}{l}\text { Polygonaceae } \\
\text { Rumex cyprius } \mathrm{L} .\end{array}$ & $\mathrm{T}$ & IT & $*$ & \\
\hline Rumex vesicarius $\mathrm{L}$. & $\mathrm{He}$ & IT.SS & & \\
\hline Rumex arvensis $\mathrm{L}$. & $\mathrm{T}$ & IT & & \\
\hline $\begin{array}{l}\text { Podophyllaceae } \\
\text { Bongardia chrysogonum (L.)Boiss. }\end{array}$ & $\mathrm{T}$ & IT,M & $*$ & \\
\hline $\begin{array}{l}\text { Primulaceae } \\
\text { Anagallis arvensis L. }\end{array}$ & $\mathrm{T}$ & COSM & $*$ & \\
\hline $\begin{array}{l}\text { Ranunculaceae } \\
\text { Anemone biflora DC. }\end{array}$ & $\mathrm{T}$ & IT,SS & & \\
\hline Ceratocephala testiculata (Crantz) Roth & $\mathrm{T}$ & IT & & \\
\hline Ranunculus arvensis L & $\mathrm{T}$ & IT & & \\
\hline $\begin{array}{l}\text { Resedaceae } \\
\text { Reseda lutea } \mathrm{L} .\end{array}$ & $\mathrm{T}$ & IT,SS & $*$ & $*$ \\
\hline $\begin{array}{l}\text { Rhamnaceae } \\
\text { Ziziphus spina-chirsti (L.) Willd }\end{array}$ & $\mathrm{p}$ & PL & & \\
\hline $\begin{array}{l}\text { Ziziphus nummularia (Burm. f.) Wight \&Arn. } \\
\text { Rosaceae }\end{array}$ & $\mathrm{p}$ & SS & $*$ & \\
\hline $\begin{array}{l}\text { Cerasus microcarpa subsp. Diffusa (Boiss. } \\
\& \text { Hausskn) Browicz }\end{array}$ & $\mathrm{p}$ & IT & $*$ & \\
\hline Amygdalus lycioides Spach & $\mathrm{p}$ & IT & & \\
\hline Amygdalus scoparia spach. & $\mathrm{p}$ & IT & $*$ & $*$ \\
\hline $\begin{array}{l}\text { Rubiaceae } \\
\text { Callipeltis cucullaria (L.) Stev. }\end{array}$ & $\mathrm{T}$ & IT, SS & & \\
\hline Galium setaceum $\mathrm{L}$ & $\mathrm{T}$ & IT, SS & & \\
\hline $\begin{array}{l}\text { Sinopteridaceae } \\
\text { Cheilanthes catanensis (Cosent.) H.P. Fuchs. }\end{array}$ & $\mathrm{Cr}$ & IT, M & $*$ & \\
\hline $\begin{array}{l}\text { Solanaceae } \\
\text { Datura stramonium L. }\end{array}$ & $\mathrm{T}$ & IT & & $*$ \\
\hline $\begin{array}{l}\text { Thymeleaceae } \\
\text { Daphne mucronata Royle. }\end{array}$ & $\mathrm{p}$ & IT & $*$ & $*$ \\
\hline $\begin{array}{l}\text { Valerianaceae } \\
\text { Valerianella vesicaria }(\text { L.) Moench. }\end{array}$ & $\mathrm{T}$ & IT & $*$ & \\
\hline $\begin{array}{l}\text { Zygophyllaceae } \\
\text { Peganum harmala } \mathrm{L}\end{array}$ & $\mathrm{He}$ & ES, IT, M, SS & & $*$ \\
\hline
\end{tabular}


The results showed that therophytes (65.4\%), hemicryptophytes (14.01\%), cryptophytes $(7 / 69 \%)$, chamaephytes $(7 / 1 \%)$ and phanerophytes $(5 / 8 \%)$ were the dominant life forms of the area, respectively (Table 1). The families with the highest number of species were Asteraceae (26 species) followed by Fabaceae ( 25 species), Poaceae (17 species), Lamiaceae (12 species), Brassicacea (10 species) and Apiaceae ( 9 species). The dominant Trees based on basal area were Quercus brantii with $26.88 \mathrm{~m}^{2}$ followed by Amygdalus scoparia with $7.2 \mathrm{~m}^{2}$. Thus, dominant families based on basal area were Fagaceae followed by Rosaceae. The dominant Trees based on relative density were Quercus brantii (48.34) followed by Amygdalus scoparia (33.1). The dominant Trees based on relative frequency were Quercus brantii (7.29) followed by Acer monspessulanum (26). The dominant Trees based on relative dominance were by Acer monspessulanum (43) followed Quercus brantii (36.45). But, the highest Trees SIV were Quercus brantii (92.08) followed by Amygdalus scoparia (47.6) (Table 2).

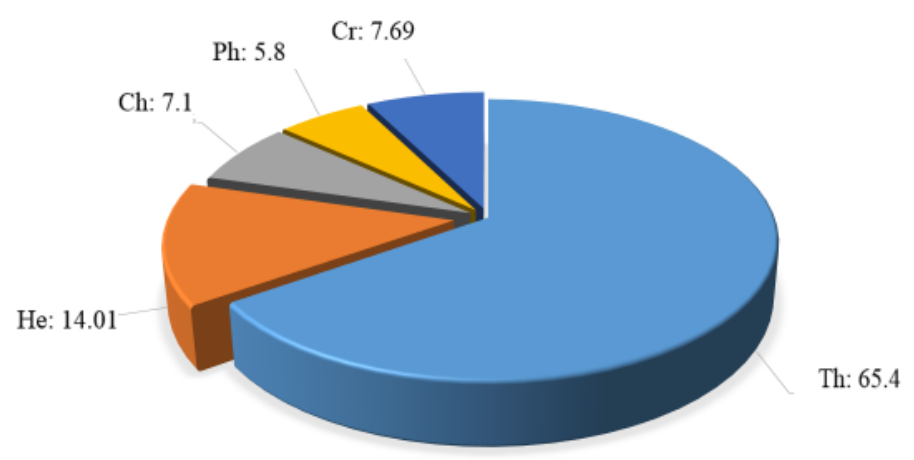

Figure 1 Life forms in the studied area, Ph: phanerophyte, Ch: chamaephytes, He: hemicryptophytes, Th:Therophytes, Gr: Cryptophytes

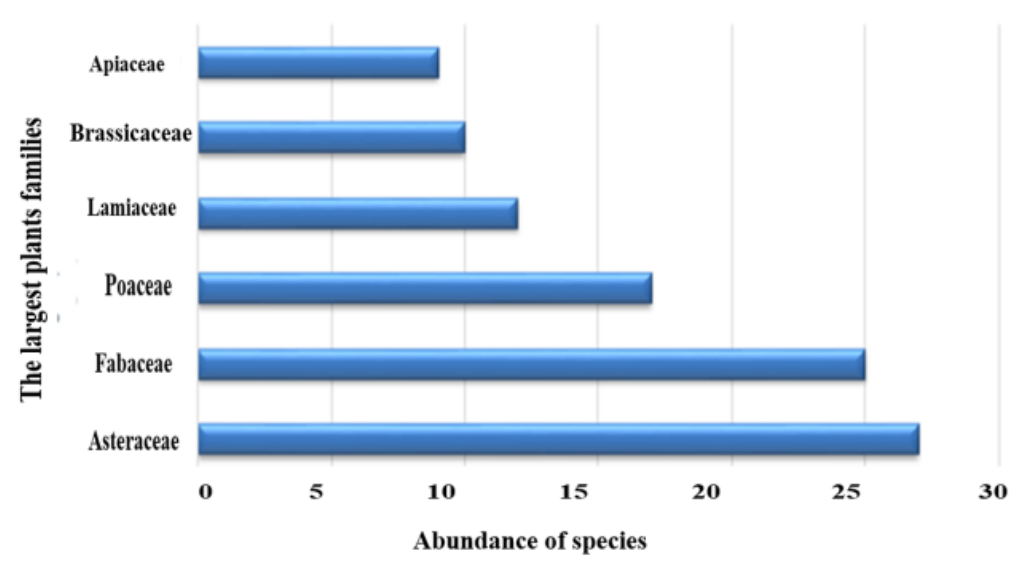

Figure 2 Abundance of plants families in the study area

Table 2 Abundance, density, frequency, basal area and Species Importance Values (SIV) of the tree layers

\begin{tabular}{lccccc}
\hline \multicolumn{1}{c}{ Tree Species } & $\begin{array}{c}\text { Basal } \\
\text { area } \\
(\mathrm{m} 2)\end{array}$ & $\begin{array}{c}\text { Relative } \\
\text { density }\end{array}$ & $\begin{array}{c}\text { Relative } \\
\text { frequency }\end{array}$ & $\begin{array}{c}\text { Relative } \\
\text { dominance }\end{array}$ & SIV \\
\hline Quercus brantii & $26 / 88$ & $48 / 34$ & $7 / 29$ & $36 / 45$ & $92 / 08$ \\
Pistacia atlantica & $1 / 92$ & $7 / 8$ & $1 / 13$ & $2 / 6$ & $11 / 53$ \\
Pistacia khinjuk & $0 / 39$ & $2 / 6$ & $0 / 37$ & $0 / 52$ & $3 / 49$ \\
Amygdalus scoparia & $7 / 2$ & $33 / 1$ & $4 / 79$ & $9 / 76$ & $47 / 65$ \\
Acer monspessulanum & $0 / 32$ & $1 / 8$ & $26 / 0$ & $43 / 0$ & $2 / 49$ \\
\hline
\end{tabular}


The dominant shrub layers based on relative density were Ziziphus nummularia with 95.89 followed by Daphne mucronata with 6.1. The dominant shrub layers based on relative frequency were Ziziphus nummularia with 0.81 followed by Daphne mucronata with 0.05 . Therefore, the highest shrub layers based SIV was Ziziphus nummularia with 96.7 (Table 3).

Table 3 Abundance, density, frequency, basal area and Species Importance Values (SIV) of the shrub layers

\begin{tabular}{cccc}
\hline Shrub species & Relative density & Relative frequency & SIV \\
\hline Daphne mucronata & $6 / 1$ & $0 / 05$ & $6 / 15$ \\
Cerasus microcarpa & $2 / 46$ & $0 / 02$ & $2 / 48$ \\
Ziziphus nummularia & $95 / 89$ & $0 / 81$ & $96 / 7$ \\
\hline
\end{tabular}

The dominant herbaceous layers based on relative density were Asteraceae family (26.6) followed by Fabaceae with (24.76). The dominant herbaceous layers based on relative diversity were Asteraceae family (16.6) followed by Fabaceae (15.43). The dominant herbaceous layers based on relative dominance were Asteraceae family (40.02) followed by Lamiaceae (21.66). So, the highest herbaceous layers based on FIV were Asteraceae family (83.22) followed by Fabaceae (65.22) (Table 4).

Table 4 Abundance, density, frequency, basal area and Family Importance Values (FIV) of the herbaceous species

\begin{tabular}{ccccc}
\hline Families & Relative density & Relative diversity & Relative dominance & FIV \\
\hline Asteraceae & $26 / 6$ & $16 / 66$ & $40 / 02$ & $83 / 22$ \\
Fabaceae & $24 / 76$ & $15 / 43$ & $25 / 03$ & $65 / 22$ \\
Lamiaceae & $11 / 42$ & $8 / 02$ & $21 / 66$ & $40 / 03$ \\
Poaceae & $20 / 95$ & $10 / 49$ & $16 / 61$ & $48 / 15$ \\
Brassicaceae & $9 / 52$ & $6 / 17$ & $8 / 33$ & $24 / 02$ \\
Apiaceae & $7 / 14$ & $4 / 32$ & $10 / 01$ & $21 / 47$ \\
\hline
\end{tabular}

The results indicated that there was the geometric distribution model in the tree layers (Figure 3). The most and the least SIV Quercus brantii and Pistacia khinjuk. Broken stick model was found in the shrub layer (Figure 4). The most and the least SIV were Ziziphus nummularia and Cerasus microcarpa. The normal logarithmic model was model was detected in the herbaceous layers (Figure 5). The most and the least fIV were Asteraceae and Apiaceae.

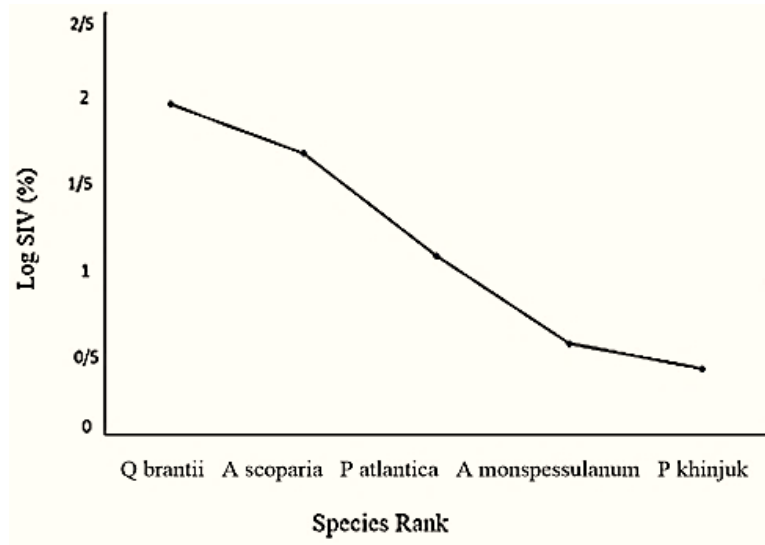

Figure 3 Distribution curve of species the in tree species

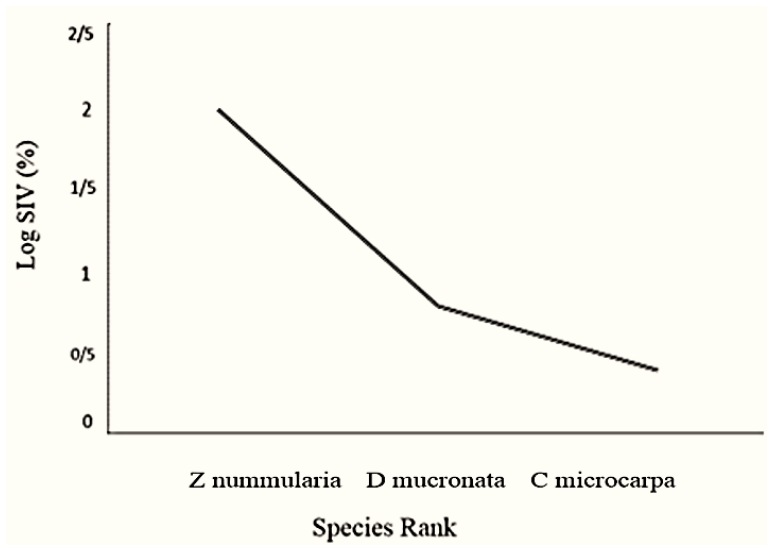

Figure 4 Distribution curve of species abundance in the shrub species 


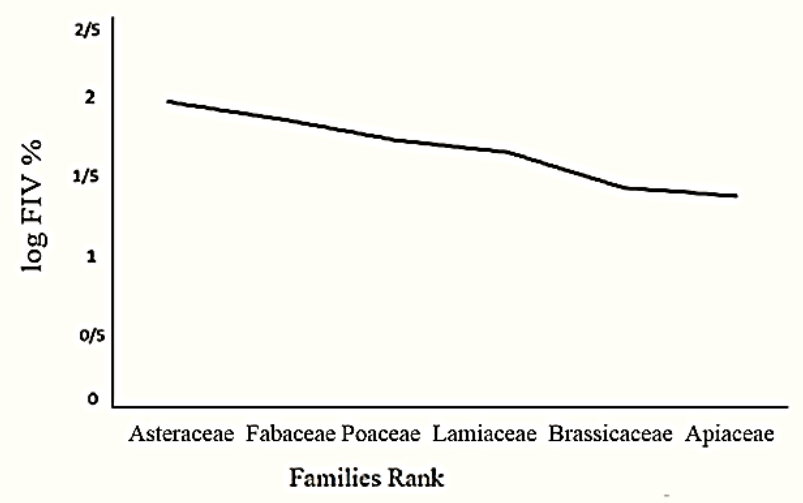

Figure 5 Distribution curve of species abundance in the families

\section{Discussion}

Land use changes act as a significant factor in the environmental changes in today's world. Unfortunately, for the last decades there has everywhere occurred a planned intrusion ofman into the deserts ecosystems. Floristic studies represent the past and present status in a region, and also play an important role in future predictions. This forest comprised of three storey (over storey: tree layers, middle storey: shrub layers, under storey: herbaceous layers). The results showed that Asteraceae and Fabaceae were the richest families in the present study. Fabaceae plays a role in soil fertility (Taber, 1973). Land use changes act as a significant factor in the environmental changes in today's world. Unfortunately, for the last decades there has everywhere occurred a planned intrusion of man into the deserts ecosystems. Floristic studies represent the past and present status in a region, and also play an important role in future predictions. The results showed that Asteraceae and Fabaceae were the richest families in the present study. Fabaceae plays a role in soil fertility (Taber, 1973) Land use changes act as a significant factor in the environmental changes in today's world. Unfortunately, for the last decades there has everywhere occurred a planned intrusion ofman into the deserts ecosystems. Floristic studies represent the past and present status in a region, and also play an important role in future predictions.

The results showed that Asteraceae and Fabaceae were the richest families in the present study. Fabaceae plays a role in soil fertility (Taber, 1973). The frequency of Asteraceae may be due to damage caused by grazing in some areas of the region which should be considered warning for the area (Negahdarsaber et al., 2017). Among identified species, 28 species have medicinal properties (Niknejad et al., 2014). The dominance of Therophytes and Hemicryptophytes can be referred to the simultaneous effects of climate fluctuations and livestock grazing on the flora of winter and rural rangelands in this area. This plants are the characteristics of the cold climate and mountainous region. High percentage of Trophytes in the region indicates two factors of degradation and drought in the region (Veiskarami et al., 2012). Therefore, this point explains the value of flora of the region. $42.5 \%$ of the species belongs to the Irano-Turanian region. High percentage of IranoTouranian elementes indicated that the area belonged to this phytochorion (Yavari and Shahgolzari, 2010).

The results showed the geometric distribution model in the tree layers. It shows that the plant communities characterized by many low species numbers can be predict an immature plant community with low biodiversity (Magurran, 2003; Pourbabaei, 2010). The presence of 5 trees species with low frequency are confirmed. The highest value for a certain species suggests that the species is dominant in the layers. Quercus brantii community with Amygdalus Scoparia and Pistacia atlantica is a one of the important communities in the Oak forest. Quercus brantii Lindl is the only present species in the Quercus genus, fagaceae family. Quercus genus has significant ecological importance in of tree communities, especially in South Zagros mountainous with one Quercus brantii species. Bischetti et al. (2007) reported that the roots of plants plays an important role in effecting soil stability and slope protection in forests, especially in mountainous areas. The broken stick model indicative a plant community with relatively uniform frequency for all the species, the number of shrubs confirmed that this issue (Magurran, 2003). Acer monspessulanum, Pistacia khinjuk and Pistacia atlantica in tree layers and Cerasus microcarpa, Daphne mucronata and Ziziphus nummularia in shrub layers were 542 
introduced as the rare species and sites have remarkable diversity measures thus it is necessary to be considered as protected sites. In particular, shrubs play the role of a nursing tree, In particular, shrubs have the role of protecting seedlings. The normal logarithmic model indicating a plant community with rich diversity where most of the species have intermediate frequency with only a few species having very high or low frequency (Hamilton, 2005; Pourbabaei, 2010). The presence of 154 herbaceous species confirms this issue. In the current research, assessment of the frequency curves in the herbaceous layers showed a normal logarithmic distribution. Destruction has been reducing the diversity of shrub and tree species and increases grass species. It is affected by different factors such as human impact, fire, climate, topography and soil on the establishment of vegetation (herbaceous layers, shrub layers) especially shrub and tree layers (Mohtashamnia et al., 2007). Studies have shown that the development of the tree layer greatly depends on the methods and intensity of forest management and and use changes act (Verburg et al., 2004).

\section{CONCLUSION}

According to results of this study, this region is being destroyed as part of Malé Galle protected area. Therefore, plant studies will be high value, and changes are considered as a serious warning. Study on flora and geographical origin of vegetation are one of the most effective methods for conservation and management of biodiversity and sustainable forest management. For example: supporting livelihood in local communities, training programs and raising awareness of local people. In its broadest sense, sustainable forest management encompasses the administrative, legal, technical, economic, social and environmental aspects of preservation. Thus, SIV and FIV are introduced as a one of the most important indexes in forest management and the index can be useful in biodiversity conservation.

\section{REFERENCES}

[IUCN] International Union for Conservation of Nature and Natural Resources. 1976. Proceedings of an International Meeting on Ecological Guidelines for the Use of Natural Resources in the Middle East and South West Asia; 1975 May 24-30; Persepolis, Iran.

Asaadi AM. 2009. Floristic study of Firozeh watershed-north Khorasan Province. Research Journal of Biological Sciences. 4(10): 1092-1103.

Bischetti GB, Chiaradia EA, Simonato T, Speziali B, Vitali B, Vullo P, Zocco A. 2007. Root strength and root area ratio of forest species in Lombardy (Northern Italy). Plant and Soil. 278: 11-22.

Farzam M, Melati F, Atashgahi. 2011. Flora, life form and chorology of winter and rural range plants in the Northern Khorasan Province, Iran. Journal of Rangeland Science. 1(4): 269-282.

Hamilton AJ. 2005. Species diversity or biodiversity?. Journal of Environmental Management. 75: 89-92.

Heinrich M, Barnes J, Gibbons S, Williamson EM. 2004. Fundamentals of Pharmacognosy and Phytotherapy. London (GB): Churchill Livingstone.

Körner C, Basler D. 2010. Phenology under global warming. Science. 327(5972): 1461-1462.

Nadaf M, Ejtehadi H, Mesdaghi M, Farzam M. 2017. Flora, life form and chorology of plants in JozakChaminbid area, North Khorassan Province, Iran. Iranian Journal of Plant Biology. 4(32): 69-88.

Negahdarsaber MR, Abkenar KT, Pourbabaei H, Sagheb-Talebi K. 2017. Flora, life forms and chorology of plant species in the Deh-Kohneh Forest in Sepidan, Fars Province, Iran. Caspian Journal of Environmental Sciences. 15(1): 67-74.

Noroozi J, Akhani H, Breckle SW. 2008. Biodiversity and phytogeography of the alpine flora of Iran. Biodiversity and Conservation. 17(3): 493-521.

Niknejad Y, Rezaee MB, Zakerimehr MR. 2014. Florestic investigation, life form, and distribution of medicinal plants species in Rineh area Amol. Eco-Phytochemical Journal of Medical Plants. 1(4): 3243. 
Magurran AE, Henderson PA. 2003. Explaining the excess of rare species in natural species abundance distributions. Nature. 422(6933): 714.

Moradipour L, Babaei H, Hatami A. 2018. Vegetation classification in relation to environmental factors, in Fars Province, Iran. Journal of Biological Studies. 1(2): 49-58.

Mohtashamnia S, Zahedi G, Arzani H. 2007. Vegetation ordination of step pic rangelands in relation to edaphical and physiographical factors (case study: Abadeh rangelands, Fars). Rangeland. 1(2): 142-158.

Moradkhani S, Milan BS. 2015. Floristic study of the rangeland Gugerd region in Khoy city (West Azarbaijan Province, NW Iran). Journal of Biodiversity and Environmental Sciences. 6(6): 48-59.

Pourbabaei H, Abedi R. 2013. Plant species groups in chestnut (Castanea sativa Mill) sites, Hyrcanian Forests of Iran. Ecologia Balkanica. 5(1): 37-47.

Parmesan C. 2007. Influences of species, latitudes and methodologies on estimates of phenological response to global warming. Global Change Biology. 13(9): 1860-1872.

Parmesan C, Yohe G. 2003. A globally coherent fingerprint of climate change impacts across natural systems. Nature. 421(6918): 37.

Pourbabaei H. 2010. Application of Statistics in Ecology (Methods and basic calculations). Rasht (IR): University of Gilan.

Perry AL, Low PJ, Ellis JR, Reynolds JD. 2005. Climate change and distribution shifts in marine fishes. Science. 308(5730): 1912-1915.

Razavi SM, Mattaji A, Rahmani R, Naghavi F. 2012. The assessment of plant species importance value (SIV) in Beech (Fagus orientalis) forests of Iran (A case study: Nav district 2 of asalem, Guilan Province). Int Res J App Basic Sci. 3(2): 433-439.

Rios JL, Recio MC. 2005. Medicinal plants and antimicrobial activity. Journal of Ethnopharmacology. 100(12): 80-84.

Sarmiento G, Monasterio M. 1983. Life forms and phenology. Ecosystems of the World. 13: 79-108.

Sharma N, Kant S. 2014. Vegetation structure, floristic composition and species diversity of woody plant communities in sub-tropical Kandi Siwaliks of Jammu, J \& K, India. International Journal of Basic and Applied Sciences. 3(4): 382.

Smith WG. 1913. Raunkiaer's "life-forms" and statistical methods. Journal of Ecology. 1(1):16-26.

Taber S. 1973. Influence of pollen location in the hive on its utilization by the honeybee colony. Journal of Apicultural Research. 12(1): 17-20.

Verburg PH, Schot PP, Dijst MJ, Veldkamp A. 2004. Land use change modelling: current practice and research priorities. GeoJournal. 61(4): 309-324

Valipour A, Namiraninan M, Etemad V, Ghazanfari H. 2009. Relationships between diameter, height and geographical aspects with bark thickness of Lebanon oak tree (Quercus libani Oliv.) in Armardeh, Baneh (Northern Zagros of Iran). Research Journal of Forestry. 3(1): 1-7.

Vaseghi P, Ejtehadi H, Zokaii M, Joharchi MR. 2007. Study of floristics, vegetation structure and chorology of plants in Kalat highlands of Gonabad, Khorasan Province, east of Iran. Journal of Science of Tarbiat Moalem University. 8: 75-88.

Veiskarami Z, Pilehvar B, Soosani, J, Veiskarami GH, Zeinivand H. 2012. Study of flora, life form and chorology of perk forest in Lorestan province, Iran. Natural Ecosystems of Iran. 3(1): 27-28.

Yavari A, Shahgolzari SM. 2010. Floristic study of Khan-Gormaz protected area in Hamadan Province, Iran. Int J Agric Biol. 12: 271-275. 